\title{
Considerations regarding the use of infant formula products in infant and young child feeding in emergencies (IYCF-E) programs
}

Authors:

Karleen Gribble1, Christine Fernandes ${ }^{2}$

Author affiliations:

1. School of Nursing and Midwifery, Western Sydney University, Parramatta, NSW. Australia.

2. Save the Children International

Correspondence to:

Karleen Gribble, School of Nursing and Midwifery, Western Sydney University, Locked Bag 1797, Penrith, NSW. 2751. Australia.

email: K.Gribble@westernsydney.edu.au

tel: $\quad+61(0) 431118485$

Competing interests The authors declare that they have no conflicts of interest. Neither have ever received research, consultation, writing or speaking funds or in-kind assistance from companies that manufacturer breastmilk substitutes.

Funding This study was self-funded with no external source of funds.

\section{Acknowledgements}

The authors would like to thank Marie McGrath, Patti Rundall, Isabelle Modigell and the anonymous reviewers for their helpful comments and assistance in the development of this paper. 


\section{Abstract}

Infants and young children are vulnerable in emergencies. The Operational Guidance on Infant and Young Child Feeding in Emergencies (OG-IFE) provides direction to governments, aid organisations and individuals on how to support the wellbeing of this population. The importance of breastfeeding in promoting child survival in emergencies cannot be overestimated. Therefore, the primary intervention in infant and young child feeding in emergencies (IYCF-E) is to support exclusive breastfeeding for infants 0-6 months and then continued breastfeeding alongside complementary feeding for children 6-24 months. However, non-breastfeeding children are vulnerable in emergencies and require intensive support. The OG-IFE outlines requirements and protocols to minimise the risks of artificial feeding in emergencies. This paper explains the reasoning behind, and the application of, these requirements. The OG-IFE emphasises that infant formula should not be provided unless breastfeeding is not possible and that except in extraordinary circumstances, individual assessment of the need for infant formula is required. It states that infant formula should be purchased and not donated and that neither follow-on formula nor toddler milks should be used. It notes that whether ready-to-use liquid infant formula or powdered infant formula is most suitable for a program will depend upon factors such as the availability of funds, of products, of water and fuel, and of storage facilities. It says that infant formula labelling should meet the requirements of the International Code of Marketing of Breastmilk Substitutes and be in the language of the recipient population, with appropriate instructions and warnings. It describes how caregivers should be discouraged from using bottles and supported to use easily cleanable cups for feeding infant formula. It notes that infant formula should be provided to infants unable to access breastmilk until they are at least 6 months of age. It also says that caregivers of formula-fed infants should be supported with a package of other resources to support safer feeding that includes: fuel, clean water, equipment for household preparation and feeding of infant formula, one-to-one education and demonstrations on safe preparation of infant formula feeds, and growth and health monitoring of the infant. It stresses that breastfeeding support should be provided alongside any artificial feeding program so as not to displace breastfeeding. Finally, it notes that in order to maximise effectiveness, IYCF-E requires a cross sectorial response with the involvement of various sectors, including child protection, water sanitation and hygiene (WASH), health, HIV, mental health and psychosocial support, education, disability, logistics, shelter, food security and livelihoods, and cash transfer programs. 


\section{Introduction}

Infants and young children are vulnerable in any emergency. Their high susceptibility to infection, inability to care for themselves and extremely specific food and fluid needs mean that they require special attention in emergency response. In particular, they require aid that supports their hydration and nutritional needs. The importance of breastfeeding in maximising child survival in emergencies should not be underestimated. Breastfeeding provides children with a source of safe food and water as well a multitude of anti-infective agents that help to prevent and fight infection. Therefore, the primary intervention in infant and young child feeding in emergencies (IYCF-E) is to support exclusive breastfeeding for infants 0-6 months and then continued breastfeeding alongside complementary feeding for children 6-24 months (IFE Core Group 2017).

However, where there are infants who cannot be breastfed or gain access to breast milk, interventions to support the non-breastfed child with infant formula are required (IFE Core Group 2017). Ensuring that artificial feeding is carried out with an adequate level of safety is difficult, but support is critical as nonbreastfed infants experience a heightened risk of infection and death in emergencies (e.g. Arvelo et al. 2010). Recent emergencies involving large numbers of partially or fully infant formula dependent infants, such as the Ukraine conflict in 2015, the European Refugee Crisis in 2015-16 and the Syrian conflict in 2011-2018, have posed particular challenges to those providing humanitarian assistance (Mboya 2014, UNICEF 2017, Summers and Bilukha 2018).

Organisations and individuals with a history of supporting infant and young child feeding (IYCF) in either development or emergency contexts are often best placed to implement artificial feeding programs. However, there can be reluctance to do so because of concerns that provision of infant formula will undermine and displace breastfeeding. This concern is valid if agencies provide infant formula without adhering to the international guidelines and protocols. However, as will be described, when artificial feeding programs are properly implemented, breastfeeding support is integrated within the program. This supports not only the health of non-breastfed infants but has resulted in increased rates of breastfeeding and reduced rates of artificial feeding in past emergencies. In contrast, not implementing an artificial feeding program leaves a gap which is often filled by organisations and individuals inexperienced in IYCF. Such organisations and individuals often engage in practices, such as untargeted distributions of infant formula, that undermine breastfeeding and place both breastfed and non-breastfed infants at risk. Properly implemented artificial feeding programs should be considered as protective of breastfed infants as well as of infants who cannot be breastfed.

Guidance on supporting the non-breastfed infant is available in the Operational Guidance on Infant and Young Child Feeding in Emergencies (OG-IFE) ${ }^{1}$; an updated version of which was published in September, 2017 (IFE Core Group

\footnotetext{
1 The OG-IFE was endorsed by the World Health Assembly in WHA 63.23 and WHA 71.9 It is available at this link: https://www.ennonline.net/operationalguidance-v3-2017
} 
2017). However, actors new to IYCF-E have a steep learning curve in implementing artificial feeding programs in emergencies and in understanding the nuance and reasoning behind aspects of this international guidance. With regards to the use of infant formula in emergencies, areas that have proved difficult to understand for those new to IYCF-E include the recommendations and considerations concerning the type of infant formula products that might be used in IYCF-E programs, how the infant formula is procured and distributed, and the type of support that should be provided alongside any provision of infant formula. This paper explains the reasoning behind, and the application of, the OG-IFE in relation to infant formula and artificial feeding programs in emergencies.

\section{Type of infant formula product}

Where it is not possible for infants to be breastfed, the Global Strategy for Infant and Young Child Feeding recommends that a suitable breastmilk substitute should be used, such as an infant formula meeting Codex Alimentarius (Codex) standards (World Health Organization and UNICEF 2003). This recommendation is also supported by the World Health Organization Guiding Principles for Feeding Non-Breastfed Infants 6-24 Months and the OG-IFE (World Health Organization 2005, IFE Core Group 2017). However, there are numerous decisions to be made in determining the most suitable infant formula product for use in an IYCF-E program. These decisions include: whether to procure ready-touse infant formula (RUIF) or powdered infant formula (PIF); whether to procure infant formula or follow-on formula for children 6-12 months of age; and whether the inclusion or absence of premium ingredients is relevant in product procurement.

\section{Ready-to-use-infant formula versus powdered infant formula}

Two forms of infant formula are available for use in IYCF-E programs; ready-touse-infant formula (RUIF) and powdered infant formula (PIF) (IFE Core Group 2017). As the name suggests, RUIF is a product that can be fed directly to infants once the container is opened. It is a sterile product that does not require reconstitution with water and so requires the least preparation resources of any type of infant formula as no water or utensils for mixing are needed. Despite these advantages, there are some drawbacks to RUIF. It can be difficult to source, especially in large quantities, it is much more expensive than powdered infant formula, and, because of its bulk and weight, it is also difficult and costly to store and transport. Large volume containers of RUIF, between $200 \mathrm{ml}-1$ litre, may be more economical per volume. However, small volume, single serve packages ( $<60 \mathrm{ml}$ each) are preferred as opened packages must be discarded if not used immediately. Unfortunately, small serving size RUIF can be particularly difficult to source in bulk and are more expensive.

If RUIF is chosen for a program, education on the need to discard/dispose of opened containers of RUIF should be provided to caregivers and organisational staff. A common recommendation for disposal of excess infant formula is for the mother/caregiver, or older children to drink the milk after the infant has finished feeding. Another option is to supervise the consumption of RUIF at an on-site 
feeding area, where the infant formula is fed on the spot and excess discarded under the supervision of the agency providing the milk. Agencies supplying RUIF should ensure that their staff are aware that although RUIF is a sterile product, feeding this infant formula to exclusively breastfed infants will markedly displace and decrease the mother's breastmilk, increase the risk of infection to the infant, and will also impede recovery from infection (Gribble 2011). It is therefore important that RUIF not be provided unless breastmilk is unavailable for the infant.

PIF requires reconstitution with boiled and cooled water before it can be fed to infants. The need for reconstitution increases the resources required to safely prepare the infant formula. Access to potable water, fuel for heating, a pot for boiling and cooling water, and other equipment for reconstitution, as well as considerable time, are only some of the requirements to safely prepare and feed PIF to an infant. However, PIF is more easily sourced, is cheaper, and is more easily stored and transported than RUIF.

The choice of RUIF or PIF will depend upon the individual circumstances of the emergency (IFE Core Group 2017). Should the emergency context involve a stationary population such as with individuals within a refugee camp or in a host community and repeated access to the affected population is feasible, and clean water and fuel is available, powdered infant formula may be the more suitable option. However, if the emergency response is supporting a displaced population in rapid transit, or where there is no access to clean water and fuel for reconstitution and cleaning feeding equipment, RUIF may be a safer product. Transitioning from RUIF to PIF may be possible once the situation stabilises.

When selecting RUIF as the type of infant formula, emergency responders must not only consider the high costs, but also the quantities available, lead-time for ordering and shipment, high number of individual serving containers, and the weight of supply that caregivers are expected to carry with them (UNICEF et al. 2015, Save the Children n.d.). The container volumes of RUIF available can also impact the suitability of using RUIF over PIF. Where small volume RUIF is not available, use of large volume (i.e. greater than a single serving) RUIF poses risks when caregivers are not willing to discard excess infant formula. This also increases the cost of formula feeding. For example, in the 2010 Haiti earthquake and 2015-2016 European Refugee responses, large 1 litre containers of RUIF were oftentimes the only size available. This meant that caregivers had to be supplied with volumes that far exceeded what could be used in a single feed, and resulted in discarding and wasting a large amount of infant formula. Operation of breast milk substitute programs in emergencies are never easy, whichever type of infant formula is provided.

Concentrated liquid infant formula (CLIF) is also available in some contexts. Similar to RUIF, CLIF is produced in a sterile, liquid form. However, it requires reconstitution with previously boiled water in order to be suitable for use and is packaged in volumes for making up several individual feeds. That it only comes as a multi-serve product and that it must be refrigerated after opening or discarded, makes it singularly unsuitable for the emergency context. CLIF has an 
added disadvantage in that it can be easily mistaken for RUIF and if fed to infants in concentrated form, can cause serious dehydration and illness. Because of the risks of contamination of opened packages and being mistaken for RUIF, CLIF should not be used in IYCF-E programs (IFE Core Group 2017).

\section{Infant formula versus follow-on formula for infants 6-12 months of age}

Two types of formula product are described by the Codex for infants 6-12 months of age: infant formula and follow-on formula (Codex Alimentarius 1981, Codex Alimentarius 1987). These two types of formula product have different composition and are produced for children of different, but overlapping age ranges. Infant formula is a breastmilk substitute manufactured for children from 0 to 12 months of age (Codex Alimentarius 1981). Throughout the world, infant formula products that meet Codex standards are accepted as suitable for use as a substitute for breastmilk for infants 0-12 months. ${ }^{2}$

Follow-on formula is manufactured for children from 6-36 months of age ${ }^{3}$. Follow-on formula, as described by the Codex, is not manufactured to be a breastmilk substitute but as a liquid complementary food (Codex Alimentarius 1987). Infants $6-12$ months who are fed a follow-on formula meeting Codex standards as their main food may be at risk of nutritional deficiencies (World Health Organization 2013). However, the composition of follow-on formula varies between countries and in some markets, it is constituted and marketed as suitable for use as a breastmilk substitute for infants from 6-12 months (e.g. Australia (NHMRC 2013)). Thus, whether follow-on formula is suitable as a replacement for breastmilk or infant formula for infants between 6 and 12 months depends on the composition of the product, and the composition varies depending upon location. What is clear is that all follow-on formula is nutritionally inadequate for young infants and should not be fed to children 0-6 months of age, whereas, non-breastfed infants, including those 6-12 months, can be fed infant formula along with appropriate complementary foods.

However, the packaging, marketing and widespread promotional claims for follow-on formula have created the impression that these products should be used in preference to infant formula once infants attain 6 months of age. This has caused some confusion in IYCF-E response, with some actors unsure about whether they should procure follow-on formula for older infants in IYCF-E programs. In fact, a variety of international and national bodies have advocated that follow-on formulas are unnecessary products (World Health Assembly 1986, New Zealand Ministry of Health 2012, NHMRC 2013, World Health Organization 2013, Crawley and Westland 2015).

Rather than being created in response to a real need, there is evidence that the the product category of follow-on formula was invented in response to regulations impacting the marketing of infant formula. What constitutes ethical

\footnotetext{
2 Infant formula suitable for infants from birth is commonly called "Stage 1" infant formula.

${ }^{3}$ Follow-on formula marketed for infants 6-12 months of age is also often called "Stage 2" infant formula.
} 
and unethical practice in the marketing of substitutes for breastmilk (including infant formula) is described by the World Health Organization's International Code of Marketing of Breastmilk Substitutes and subsequent relevant World Health Assembly resolutions (the Code) (World Health Organization 1981). The Code states that there should be no direct advertising or other promotions of breastmilk substitutes to the general public (World Health Organization 1981). As a result, many countries have legislative and other regulatory mechanisms that restrict the marketing of infant formula. In response, manufacturers have produced follow-on formula, which they market and advertise freely, claiming that such activities are allowable because the products are not infant formula (Berry et al. 2011, UNICEF 2013, World Health Organization 2013). Thus, the production of follow-on formula has enabled manufacturers to circumvent regulatory mechanisms allowing them to advertise formula products for older infants and young children. In addition, by using similar packaging and branding for follow-on formula and infant formula, any advertising of follow-on formula also indirectly promotes their infant formula (Berry et al. 2011).

Thus, although it is commonly believed that once formula-dependent infants attain 6 months of age they should be fed follow-on formula, this is not the case. Furthermore, use of follow-on formula in IYCF-E programs has significant disadvantages. First, it makes calculating the required volume of formula product more difficult and increases the probability that too little or too much product will be purchased. If too little product is purchased then the health of infants can suffer and agencies will fail in their responsibility under the Code and the OG-IFE to provide a continuity of supply of breastmilk substitutes for nonbreastfed infants in their IYCF-E programs (World Health Organization 1981, IFE Core Group 2017). The purchase of too much formula product is problematic because formula products are costly and as such, a significant expense to organisations. In addition, because infant and follow-on formula are such expensive and highly valuable commodities, especially in emergencies, individuals and organisations have great difficulty in discarding unused product. A surplus of infant or follow-on formula places pressure on agencies to distribute the remaining volume and can result in unnecessary formula feeding with attendant risks. Second, confusion about the difference between these products is not uncommon and can result in babies under 6 months being fed follow-on formula with associated increased risks. Third, ascertaining whether products are suitable for use as a breastmilk substitute for older infants can be difficult. Thus, the OG-IFE states that follow-on formula should not be used in IYCF-E programs but that infant formula be procured where artificial feeding is being supported for non-breastfed infants 6-12 months of age (IFE Core Group 2017).

So called, "growing-up" milks and toddler milks for children over a year of age are similarly unnecessary products with the same history of creation to circumvent marketing restrictions (Berry et al. 2011). They have no place in IYCF-E programs (IFE Core Group 2017). Where providing milk to children over 1 year of age is desired, unmodified animal milks can be used but care must be taken to ensure that "spillover" to breastfed infants does not occur. This can be managed by mixing milk powder with cereal to make a porridge mix suitable for use as a complementary food or by providing liquid milk for immediate on-site 
consumption (also known as wet feeding) (IFE Core Group 2017). The OG-IFE articulates that milk, whether in dried or liquid form, should never be included in general distributions of food due to the risk of it being used as a breastmilk substitute.

\section{Presence or absence of "premium" ingredients}

In any context, the price of infant formula may vary widely. The most expensive product may be as much as three times the cost of the cheapest product within the same category. Manufacturers use branding including scientific language and iconography, attractive designs with gold decoration and nutrition content claims concerning "special ingredients", amongst other strategies to suggest that their "premium", more expensive infant formula is of higher quality than the cheaper products. However, the Codex standard lists the essential ingredients that all infant formula products must contain. Thus, attractive packaging, persuasive language and nutrition content claims are not evidence of an infant formula being a higher quality product. IYCF-E programs should use infant formula that meets the Codex standard and disregard any marketing or special ingredient claims in deciding which infant formula to purchase (IFE Core Group 2017).

\section{Donation or purchase of infant formula}

In emergencies, individuals, governments, non-governmental organisations and businesses often donate infant formula. Decades of experience have shown that donations of infant formula are problematic in emergencies. They are often not provided in the amount required (too much or not enough) and on-going supply cannot be guaranteed. They are also often delivered to the wrong place, are of the wrong type, are expired or close to expiry, and labelled in the wrong language (Borrel et al. 2001, Gribble 2014, IFE Core Group 2017). Donations of infant formula have been identified as causing problems in a large number of emergencies including: the Balkan crisis of the late 1990s (Borrel et al. 2001), the second Chechen war of 2000 (World Health Organization, UNICEF et al. 2000), the 2004 Indian Ocean Tsunami in Thailand, Sri Lanka and India (Carballo and Heal 2005, Jayathilaka 2005, Adhisivam et al. 2006), the 2005 Kashmir earthquake (Arts 2006), the 2008 conflict in Georgia (UNICEF 2008), the 2009 internally displaced person crisis in Pakistan (IRIN 2009), the 2010 Haitian earthquake (Nybo 2010), the 2011 Christchurch earthquake (Bartle 2011), the Syrian civil war (Mboya 2014) and the 2015-16 European Refugee Crisis (Modigell et al. 2016) to name but a few.

In many instances, those donating infant formula are doing so because they wish to assist infants. However, emergencies are also often used by infant formula manufacturers as a promotional opportunity and as a way of cultivating new markets or growing existing ones (Gribble 2013). In addition, as is the case with pharmaceuticals, infant formula manufacturers are prone to dumping product that they have been unable to sell, including that which is close to or past expiry (Autier et al. 2002, Gribble 2014). 
Experience has also shown that where infant formula is donated, rather than purchased by organisations, problems with inappropriate distribution are common (Hipgrave et al. 2012). In contrast, where infant formula must be budgeted and tightly accounted for, there is greater accountability resulting in better targeted distribution (Tarrant et al. 2016). As will be discussed, formula feeding with an acceptable level of safety in an emergency requires a package of physical, educational and health support. However, distributions of donated infant formula are only rarely accompanied by this required support. Inappropriate distributions of infant formula increase rates of formula feeding and also increase morbidity and mortality rates in emergencies (Creek et al. 2010, Hipgrave et al. 2012). For these reasons, the OG-IFE and the Sphere Handbook: Humanitarian Charter and Minimum Standards in Humanitarian Response state that donations of infant formula should not be solicited or accepted by any organisation (The Sphere Project 2011, IFE Core Group 2017). Further, any donations that arrive in the region of an emergency should be collected together by a nominated agency and so removed from uncontrolled distribution networks (The Sphere Project 2011, IFE Core Group 2017). Thus, in order for an IYCF-E program to meet minimum standards, any infant formula required should be purchased by agencies that are working as part of the nutrition and health emergency response (IFE Core Group 2017).

\section{Labelling and branding of infant formula}

Infant formula used in IYCF-E programs must have instructions on preparation, use and storage that are in the languages used by the populations impacted by the emergency, which may be different from the local language (IFE Core Group 2017). Labels must also contain a statement on the superiority of breastfeeding, note that the product should only be used on the advice of a health worker (including trained community workers and volunteers), and warn of the danger of inappropriate preparation, storage and use (WHO 1981). As required by the Code, labels should not contain images or text that promote infant formula including: health claims, nutrition content claims, convenience of other promotional claims, or comparisons to breast milk (World Health Organization 1981, IFE Core Group 2017). Whenever possible, non-branded/generic infant formula should be used in IYCF-E programs (IFE Core Group 2017). This prevents an implied endorsement of the brand by the organisation running the program and prevents the emergency from contributing to expanding the market for that product.

Obtaining infant formula that is unbranded and/or meeting these labelling requirements is often extremely difficult. In such circumstances, relabeling infant formula with a sticker print-out adhered to the packaging can be an inexpensive solution (IFE Core Group 2017). Where it is not possible to obtain infant formula that meets these labelling requirements and relabeling has not been possible, instruction sheets with the required information can accompany the infant formula provision (IFE Core Group 2017). 


\section{Supporting feeding with bottles or cups}

In much of the world, infants are fed infant formula with a feeding bottle. However, feeding bottles are difficult to clean even in the best of circumstances. Milk remnants that remain in bottles can foster the growth of pathogenic bacteria (Agostoni et al. 2004, Redmond and Griffith 2009). In resource-poor contexts, including in emergencies, it is therefore, recommended that open cups be used to provide infant formula (World Health Organization 2004, Food and Agriculture Organization 2007, IFE Core Group 2017). Cups are preferred because they can be more easily cleaned than bottles. Infants can be fed using cups from birth if necessary, and instructions are available on how to do so. However, mothers and caregivers may require one-on-one assistance in learning how to cup feed (Food and Agriculture Organization and World Health Organization 2007). In order to encourage cup feeding over bottle feeding, some IYCF-E programs have carried out "bottle amnesties" where feeding bottles are exchanged for feeding cups. Where families are in transit, cups with solid lids (without spouts) have proven useful and distribution of bibs alongside cups supports cup feeding as it helps to mitigate against spillage during feeding. Disposable feeding cups can be used to eliminate the need to wash and sterilise cups. Paper cups may be preferred to plastic for environmental reasons.

Cup feeding may not be acceptable to all mothers and caregivers. Transitioning from bottle feeding to cup feeding is sometimes not easy, takes patience for each feed, and requires behaviour change from the caregiver and infant. Further, cup feeding while on the move, such as in a situation of rapid transit, can be difficult, messy, wasteful (which is problematic if there is limited product), and stressful for the mother and infant. In such circumstances, risk mitigation strategies such as providing education on bottle cleaning and disinfection, on-site cleaning services or, as a last resort, bottle exchanges may be necessary (IFE Core Group 2017). However, these strategies should be implemented alongside cup distribution and promotion of safer feeding with cups. Donations of feeding bottles should not be sought or accepted in emergencies and any donations that arrive should be collected together by a nominated agency and removed from distribution (IFE Core Group 2017).

\section{Resources that must be provided with infant formula}

Infant formula is only one component of what is required in order for infants to be artificially fed with an acceptable level of safety in emergencies. In addition to infant formula, IYCF-E programs supporting the non-breastfed infant need to ensure that the other necessary resources are also provided or are available. These resources include fuel, clean water, and equipment for household preparation and feeding of infant formula (such as a feeding cup, a pot for boiling water, a storage container for hygienic storage of supplies) (IFE Core Group 2017). On-going support is also required including one-to-one education and demonstrations on safe preparation and also growth and health monitoring of the infant (IFE Core Group 2017). Growth monitoring and follow-up of infants enrolled in an artificial feeding program should take place at least twice a month, and any defaulters should be traced (IFE Core Group 2017). Because stress, trauma, grief and depression can impact the ability of caregivers to feed and care 
for their infants, inclusion or referral for psychosocial support for bonding, responsive feeding and care of infants should be considered (IFE Core Group 2017). Where funding is sought for IYCF-E programs that include support for artificial feeding, grant applications should include costs for these additional resources (IFE Core Group 2017). If resources such as access to clean water and fuel cannot be provided in the community, then on-site feeding may be necessary.

In addition, it should be ensured that support for breastfeeding, including breastfeeding counselling, is provided whenever artificial feeding is being supported (IFE Core Group 2017). Such support makes it possible for women who are mixed feeding (breastfeeding and formula feeding) to increase their breastmilk supply and reduce formula milk supplements, or for caregivers to relactate, so reducing risk. Breastfeeding support also minimizes the possibility that an artificial feeding program in the community will result in infants being unnecessarily formula fed. This is because breastfeeding assistance enables women to overcome difficulties that might otherwise lead to infant formula use. Examples of difficulties that may be overcome with breastfeeding counselling and other support include: perceived low milk supply (not enough breastmilk), perceived milk quality issues, nipple pain, mastitis, unsettled infant behaviour, an ill mother or infant, family pressure to use infant formula, and the need for prescribed medication leading to a perception that breastfeeding should not occur.

Consideration should be given to providing breastfeeding women with goods of a similar value to that being provided to support formula feeding caregivers (for example additional food rations or a breastfeeding shawl) so as not to discourage breastfeeding (IFE Core Group 2017). As will be discussed, collaboration between sectors including nutrition, health and WASH (water sanitation and hygiene) is necessary in order to ensure that the mothers and caregivers of infants and young children are provided with the required feeding support (IFE Core Group 2017).

\section{Assessment of the need for infant formula}

Although the choice of infant formula product and method of feeding are critical, arguably the most important issue in IYCF-E artificial feeding programming is assessing whether an infant needs infant formula. Because of the dangers posed to artificially fed infants and the expense and difficulty of providing the necessary support for formula feeding, it is unethical for organisations to provide infant formula where it is not necessary (Gribble 2018). Infant formula should be treated more like a medicine than a food in emergencies. It is therefore a requirement of IYCF-E programs that, except in extraordinary circumstances, ${ }^{4}$

\footnotetext{
${ }^{4}$ As stated in the OG-IFE "In some emergencies, it may not be possible to meet all the provisions of the OG-IFE immediately, such as where access to those affected is limited or impossible, or capacity is lacking to deliver necessary support. In such circumstances, critical analysis by the IFE coordination authority, government, UNICEF, WHO and, where applicable, UNHCR is essential to provide contextspecific guidance on appropriate actions and acceptable compromises. Adapted programming may fall short of OG-IFE recommendations and should be temporary. The unmet needs and risks of compromised programming should be used to inform proactive advocacy for humanitarian access,
} 
assessment of the need for infant formula should be carried out at both the contextual and individual levels (IFE Core Group 2017).

At an individual level, an in-depth assessment should be conducted by a health or nutrition worker with training in breastfeeding (IFE Core Group 2017). As breastmilk substitute provision is also context-specific, assessment questions and targeting criteria should also be discussed with the IFE coordination authority to better understand the required benchmarks for artificial feeding.

The OG-IFE provides indications for short-term provision of infant formula which include:

- to supplement maternal milk supply while relactation ${ }^{5}$ proceeds,

- during the transition from mixed feeding to exclusive breastfeeding,

- while a wet nurse is identified,

- where a short term separation of mother and child is necessary (IFE Core Group 2017).

If an infant is not breastfed, attempts should be made to provide breastmilk. If the mother is not lactating, relactation should be explored (IFE Core Group 2017). Identifying a wet nurse or source of expressed donor milk are other options that may enable the infant to be breastfed or breastmilk fed (IFE Core Group 2017).

The indications for long-term provision of infant formula include situations where:

- the infant was not breastfed prior to the emergency and the mother is unwilling to relactate,

- the mother is HIV positive and the infant was formula fed prior to the emergency,

- rarely occurring maternal or infant medical conditions preclude breastfeeding,

- the infant is rejected or motherless, or the mother is a sexual assault survivor not wishing to breastfeed (IFE Core Group 2017).

The indication for provision of infant formula to HIV-positive women deserves some explanation. Although HIV can be transmitted via breastmilk, exclusive breastfeeding and the use of antiretroviral medication reduces transmission to an extremely low level. In the interests of HIV-free child survival, in countries with high infant mortality rates, national governments recommend that HIVpositive women exclusively breastfeed for 6 months and then continue breastfeeding for at least 12 months, all the while taking antiretroviral medication (ARV) (World Health Organization, UNAIDS et al. 2010). Where mothers and caregivers are not breastfeeding, relactation is a way of enabling provision of breastmilk. However, unless there is a wet nurse to assist in the

resourcing and capacity.”

${ }^{5}$ Relactation is the process by which woman who is not lactating is able to make milk through the suckling of a baby at the breast. The transition from no milk to a full milk supply usually takes a number of weeks. 
relactation process, relactation requires a period of mixed breastfeeding and formula feeding. Mixed feeding increases the risk of transmission of HIV but if women have access to and are able to adhere to ARVs, the risk of transmission remains low and relactation may be considered (World Health Organization 2016, World Health Organization and UNICEF 2018). In contrast, in circumstances where antiretrovirals are not available, mixed feeding associated with relactation poses a high risk of HIV transmission. The indicator for provision of infant formula for HIV positive women, is therefore in recognition of the risks of mixed feeding in relactation and the fact that access to ARVs is often interrupted during emergencies.

Breastfeeding women often request supplies of infant formula even though they are breastfeeding. They do so for a variety of reasons, including that they believe that they do not have enough milk or that infant formula is superior to breastmilk, because it is supported by cultural beliefs, because formula feeding is an aspirational activity, and because infant formula is a product of high economic value.

It is common for women to genuinely believe that they are unable to breastfeed because of the emergency. The idea that stress or lack of food prevents milk production or reduces its quality is strongly held in many cultures but it is also often based on mothers' interpretation of their infants' behaviour. While neither stress nor moderate malnutrition affects breast milk production or quality (Prentice et al. 1983, Hill et al. 2005), stress can delay the milk ejection reflex, resulting in infants becoming fussy at the breast. Mothers can interpret this as indicating that they do not have enough milk for their infants or that their milk is of poor quality. Psychosocial and breastfeeding support can ease stress and build confidence, enabling mothers to continue exclusive and continued breastfeeding (UNICEF 2008). If a woman is severely malnourished to the point that her milk supply is impacted, adequate feeding of the mother will increase her milk supply.

However, it is also important to note that the circumstances of the emergency, for example being in transit, a lack of privacy, or burdensome survival demands, can result in women breastfeeding less frequently than usual. This can result in a real decline in milk supply. Providing practical support, such as priority access to resources, as well as breastfeeding support, enables more frequent breastfeeding and will increase milk supply.

Cultural beliefs around infant feeding may result in maternal requests for infant formula. For example, in Haiti after the 2010 earthquake some believed that the experience of trauma would mean that their breastmilk was bad for their infants (Dörnemann and Kelly 2013). Therefore mothers who were distressed or traumatised requested infant formula because they did not wish to harm their infant by breastfeeding. Similarly, in many cultures colostrum is considered unhealthy for newborns and women may seek infant formula to feed their babies until their milk "comes in" (Patel et al. 2015). Education and breastfeeding support can enable women to adapt their understanding of these situations, to exclusively breastfeed their young infants, and continue to breastfeed alongside 
the introduction of complementary foods (UNICEF 2008, Dörnemann and Kelly 2013).

In many middle- and high-income countries, formula feeding has become normalised and families commonly have the expectation that they will partially or fully formula feed their babies. Some segments of the population have no cultural experience with breastfeeding and may view it as repugnant. In an emergency, mothers and family members may need assistance to understand how the emergency conditions may have changed the risk profile of, and work involved in, formula feeding and will require support to be able to breastfeed.

An aspiration to formula feed may lead caregivers to request infant formula. In many low- and in some middle-income countries, formula feeding is a practice that is undertaken by the wealthier, higher educated and high status members of society. Advertising that suggests that feeding a baby infant formula leads to improved brain function and educational and economic success has also impacted community beliefs about the desirability of formula feeding (Pries et al. 2016). When families become aware that aid organisations are distributing infant formula, it is sometimes seen as an opportunity to provide their infants with something that they have always wanted to give them but have not been able to afford (Borrel et al. 2001).

Appropriate support for breastfeeding enables women who have these beliefs to be able to continue breastfeeding, and as previously stated, artificial feeding programs in IYCF-E should be accompanied by appropriate breastfeeding support for the target population. However, the level of one-to-one feeding assessments and support may depend on the amount of time a health worker has with a caregiver. A mother and her infant in a rapid transit context may have less time for a full feeding assessment than a mother in a more stationary context, and there may not be any follow-up sessions, in which case support for formula feeding requires serious consideration before distribution (IFE Core Group 2015).

At agency level, organisations considering whether to implement a breastmilk substitute programme should consider: the national policies surrounding infant feeding; access to the target population; pre-crisis infant feeding practices and how they have changed during the emergency such as the numbers of mixed-fed infants vs non-breastfed infants, and the knowledge, beliefs and attitudes surrounding international IYCF recommendations; child nutrition status; the accessibility of infant formula; the level of capacity of IYCF-E skilled support; the WASH resources and availability of clean water; the price of fuel; and unsolicited donations of infant formula, and what other organizations working in the area are doing (IFE Core Group 2017). Ideally, a representative survey should be conducted, but when this is not possible, opportunistic means to gather data should be used such as key informant interviews, focus group discussions, rapid screenings, and/or transect walks (IFE Core Group 2017).

Each of these factors interact with one another and a decision should be made based on the full picture. An example of where a decision was made not to 
implement a breastmilk substitute program occurred in early 2018 in South Syria. Following numerous requests by mothers for infant formula, a responding international aid agency conducted an IYCF representative survey. The results showed that $98 \%$ of all caregivers were mixed breastfeeding and artificially feeding their infants. Infant formula was available in the community and breastfeeding difficulties were identified as the main reason for mixed feeding and infant formula requests. Support for breastfeeding overwhelmingly outweighed the need to provide infant formula and in this case the agency implemented a breastfeeding support program and did not implement a breastmilk substitute program.

An example of where a decision was made to implement a breastmilk substitute program occurred in the Za'atari refugee camp in Jordan in 2012. In Za'atrai, there was also a very high demand for infant formula. This demand arose because artificial feeding of infants had been common before the crisis, mothers believed that stress prevented breastfeeding, and unsolicited donation of infant formula was widespread. Upon arrival at Za'atari camp, many infants were not breastfed and many had never breastfed. In the absence of a breastmilk substitute program, there was pressure for blanket distributions of infant formula. There were also problems with parents and caregivers selling the donations of infant formula that they had received. With the presence of a comprehensive breastfeeding support program in the camp and the capacity to provide intensive support for IYCF-E, Save the Children Jordan, made the decision to implement a breast milk substitute programme. The camp context and close collaboration with midwives allowed for a highly monitored and supported artificial feeding component to the IYCF-E program. The presence of the artificial feeding program, reduced donations of infant formula and reduced their impact. The tight inclusion criteria meant that relatively few infants required artificial feeding support while the extensive support for breastfeeding maximised exclusive breastfeeding.

\section{Age of infants}

The OG-IFE states that infant formula should be provided to non-breastfed infants in IYCF-E programs for as long as children need it, that being until infants are at least 6 months of age (IFE Core Group 2017). However, in much of the world, health authorities state that that non-breastfed infants should be fed infant formula until 12 months of age (Baker and Greer 2010, NHMRC 2013, Crawley and Westland 2015). This may not be feasible or preferable in the emergency context. For example, in the European refugee crisis of 2015-16 it was deemed that the rapid transit of the refugee population meant that giving older infants pasteurized animal milks or UHT milk or yoghurt might be safer than providing powdered infant formula (IFE Core Group 2015). More commonly, organisations may simply not have the budget or resources to support formula feeding for older infants and therefore focus their attention on the younger, more vulnerable infants.

Where infant formula is not being provided for non-breastfed infants 6-12 months, pasteurised or boiled cows' milk (or another animal milk) may be used. 
However, there are a number of concerns associated with feeding whole cow's milk to infants. These concerns are associated with a heightened risk of suboptimal nutrient intake, iron deficiency anaemia, and a high solute load impacting the kidneys (Committee on Nutrition 1992). In order to mitigate these risks, WHO recommends that infants be fed nutrient rich complementary foods once a child reaches 6 months of age to avoid nutrient deficiencies, that iron supplements be added to the infant's diet to avoid anaemia in particular, and sufficient additional fluid intake be given to counter high renal solute load (World Health Organization 2005). However, it should be noted, that any complementary foods provided in IYCF-E programs should not be donated, but locally made or purchased (IFE Core Group 2017) ${ }^{6}$.

In decision making concerning whether infant formula will be provided for nonbreastfed infants 6-12 months of age, the risks of not supporting these infants in the particular context needs to be considered. For example, if there is limited access to whole cow's milk (or other animal milks) or appropriate complementary foods, this will heighten the risk of not supporting older infants with infant formula. Or, if there is controlled, long-term access to infants and their caregivers that enables repeated support visits such as in a refugee camp in a host country, this context may allow for provision of infant formula up to the age of 12 months. Pre-emergency practices as well as government and agency policy also need to be considered (IFE Core Group, 2017). Where infant formula is provided for infants ages 0-6 months, agencies must be prepared for extra supply to "wean" the infant gradually from full provision onto other milks or foods. The amount needed will depend on each particular infant and context.

\section{Cross cutting issues in artificial feeding programs in IYCF- E}

While IYCF-E is primarily a nutrition intervention, it is also a true cross-sectorial issue that requires the involvement of a large variety of sectors to successfully support the wellbeing of infants and young children (IFE Core Group 2017). Some examples of ways in which sectors other than nutrition should be involved in IYCF-E artificial feeding programs include:

- $\quad$ Child protection- Ensure that infants and young children are targeted for identification in child protection programs and that mixed fed and nonbreastfed infants will be recognised as in need of urgent referral to IYCF-E programs.

- $\underline{\text { WASH}}$ - Ensure that WASH provider agencies secure priority access of families with infants using infant formula to WASH services and enable access to cleaning equipment and advice on hygienic preparation and

\footnotetext{
6 Donated complementary foods are problematic because they may not meet nutritional and safety standards, are often inappropriately labelled (in particular they may be erroneously labelled as being suitable for feeding infants from 4 months of age), may be culturally inappropriate; and may undermine local food use and recommended IYCF practices (IFE Core Group 2017). As with infant formula donations, companies may seek to use donations of complementary foods as a way of entering or broadening markets.
} 
storage of supplies.

- $\quad$ Health- Ensure that the caregivers of partially or fully artificially fed infants receiving medical treatment for infections or malnutrition receive counselling and support by staff trained in IYCF to increase milk supply and relactate if appropriate.

- HIV- Refer HIV positive women who are artificially feeding their infants to IYCF-E programs.

- Mental Health and Psychosocial Support- Ensure that the mothers/caregivers of non-breastfed infants are identified and recognised as being in particular need of psychosocial support and are referred to IYCF-E programs.

- Disability- Ensure that the caregivers of non-breastfed fed disabled infants and disabled caregivers of artificially fed infants are referred to IYCF-E programs.

- Logistics- Have policies preventing the transport and distribution of donations of infant formula, other milks and infant feeding bottles and report donations to UNICEF or UNHCR as appropriate, and to the IYCF-E coordination authority

- Shelter- Ensure adequate conditions for the safe preparation of infant formula feeds are provided to households with a formula fed infant.

- $\quad$ Food security and livelihoods- Ensure that livelihood programmes provide day care arrangements and feeding breaks for the caregivers of infants, including those being artificially fed.

- Cash transfer programmes- Where vouchers are used for indirect provision of infant formula ensure that products at the designated vendors are meet the appropriate standard, including regarding labelling. Further detail on cross-sectorial issues in IYCF-E is provided in the UNHCR/Save the Children document, "Infant and Young Child Feeding in Refugee Situations: A Multi-Sectorial Framework for Action" (UNHCR and Save the Children 2017).

\section{Conclusions}

In an emergency, protecting, supporting and promoting safe and appropriate infant and young child feeding to both the breastfed and non-breastfed child must be prioritised to safeguard the health and wellbeing of infants and young children. However, supporting artificial feeding in emergencies is a complex area of humanitarian aid. All individuals or organisations wishing to be involved in IYCF-E should be familiar with the OG-IFE and committed to applying the guidance. 


\section{References}

Adhisivam, B., S. Srinivasan, M. B. Soudarssanane, S. Deepak Amalnath and A. Nirmal Kumar (2006). "Feeding of infants and young children in tsunami affected villages in Pondicherry." Indian Pediatrics 43(8): 724-727. https://www.indianpediatrics.net/aug2006/724.pdf

Agostoni, C., I. Axelsson, O. Goulet, B. Koletzko, K. F. Michaelsen, J. W. Puntis, J. Rigo, R. Shamir, H. Szajewska, D. Turck, Y. Vandenplas, L. T. Weaver and ESPAGHAN Committee on Nutrition (2004). "Preparation and handling of powdered infant formula: a commentary by the ESPGHAN Committee on Nutrition." Journal of Pediatric Gastroenterology and Nutrition 39(4): 320-322.

Arts, M. (2006). "Infant feeding in the South Asia aftermath." Field Exchange 27: 2-4.

https://pdfs.semanticscholar.org/6e59/d6404d73b18b27f844e64da42e9f05e0 067d.pdf

Arvelo, W., A. Kim, T. Creek, K. Legwaila, N. Puhr, S. Johnston, J. Masunge, M. Davis, E. Mintz and A. Bowen (2010). "Case-control study to determine risk factors for diarrhea among children during a large outbreak in a country with a high prevalence of HIV infection." International Journal of Infectious Diseases 14(11): e1002-1007.

https://www.sciencedirect.com/science/article/pii/S1201971210024574

Autier, P., R. Govindaraj, R. Gray, R. Lakshminarayanan, H. G. Nassery and G. Schmets (2002). Drug Donations in Post-Emergency Situations. Washington, The World Bank. https://openknowledge.worldbank.org/handle/10986/13673

Baker, R. D. and F. R. Greer (2010). "Diagnosis and prevention of iron deficiency and iron-deficiency anemia in infants and young children (0-3 years of age)." Pediatrics 126(5): 1040-1050.

http://pediatrics.aappublications.org/content/126/5/1040

Bartle, C. (2011). "Infant feeding in emergencies: Reflections on the Christchurch earthquake." Midwifery News 61: 31-32.

Berry, N. J., S. C. Jones and D. Iverson (2011). "Circumventing the WHO Code? An observational study." Archives of Disease in Childhood.

http://ro.uow.edu.au/cgi/viewcontent.cgi?article=2228\&context=hbspapers

Borrel, A., A. Taylor, M. McGrath, A. Seal, E. Hormann, L. Phelps and F. Mason (2001). "From policy to practice: Challenges in infant feeding in emergencies during the Balkan crisis." Disasters 25(2): 149-163.

https://doi.org/10.1111/1467-7717.00167

Carballo, M. and B. Heal (2005). "The public health response to the tsunami." Forced Migration Review July: 12-14. http://www.fmreview.org/tsunami/carballo-heal.html 
Codex Alimentarius (1981). Standard for infant formula and for special medical purposes intended for infants- Codex Stan 72-1981. http://www.fao.org/faowho-codexalimentarius/shproxy/fr/?lnk=1\&url=https\%253A\%252F\%252Fworkspace.fao.org\%252Fsites \%252Fcodex\%252FStandards\%252FCODEX\%2BSTAN\%2B721981\%252FCXS_072e.pdf

Codex Alimentarius (1987). Codex standard for follow-up formula- Codex Stan 156-1987. http://www.fao.org/fao-who-codexalimentarius/shproxy/en/?lnk=1\&url=https\%253A\%252F\%252Fworkspace.fao.org\%252Fsites \%252Fcodex\%252FStandards\%252FCODEX\%2BSTAN\%2B1561987\%252FCXS_156e.pdf

Committee on Nutrition (1992). "The use of whole cow's milk in infancy." Pediatrics 89(6): 1105-1109.

http://pediatrics.aappublications.org/content/pediatrics/89/6/1105.full.pdf

Crawley, H. and S. Westland (2015). "Infant milks in the UK: A practical guide for health professionals." First Steps Nutrition Trust.

https://www.universitybarclay.com/wpcontent/uploads/2016/04/Infant_milks.pdf

Creek, T. L., A. Kim, L. Lu, A. Bowen, J. Masunge, W. Arvelo, M. Smit, O. Mach, K. Legwaila, C. Motswere, L. Zaks, T. Finkbeiner, L. Povinelli, M. Maruping, G. Ngwaru, G. Tebele, C. Bopp, N. Puhr, S. P. Johnston, A. J. Dasilva, C. Bern, R. S. Beard and M. K. Davis (2010). "Hospitalization and mortality among primarily nonbreastfed children during a large outbreak of diarrhea and malnutrition in Botswana, 2006." Journal of Acquired Immune Deficiency Sydrome 53(1): 14-19. doi: 10.1097/QAI.0b013e3181bdf676

Dörnemann, J. and A. H. Kelly (2013). "'It is me who eats, to nourish him': a mixed-method study of breastfeeding in post-earthquake Haiti." Maternal \& Child Nutrition 9(1): 74-89. doi: 10.1111/j.1740-8709.2012.00428.x.

Food and Agriculture Organization and World Health Organization (2007). How to Prepare Formula for Cup-Feeding at Home. Geneva, World Health Organization. http://www.who.int/foodsafety/document_centre/PIF_Cup_en.pdf

Gribble, K. D. (2011). "Mechanisms behind breastmilk's protection against, and artificial baby milk's facilitation of, diarrhoeal illness." Breastfeeding Review 19(2): 19-26.

Gribble, K. D. (2013). "Media messages and the needs of infants and young children after Cyclone Nargis and the WenChuan Earthquake." Disasters 37(1): 80-100. doi: 10.1111/j.1467-7717.2012.01289.x 
Gribble, K. D. (2014). Formula feeding in emergencies. Handbook of Dietary and Nutritional Aspects of Bottle Feeding. V. R. Preedy, R. R. Watson and S. Zibadi. Wageningen. The Netherlands, Wageningen Academic Publishers: 143-161.

Gribble, K. D. (2018). "Supporting the most vulnerable through appropriate infant and young child feeding in emergencies " Lournal of Human Lactation 34(1): 40-46.

http://journals.sagepub.com/doi/abs/10.1177/0890334417741469?journalCo de $=$ jhla

Hill, P. D., J. C. Aldag, R. T. Chatterton and M. Zinaman (2005). "Psychological distress and milk volume in lactating mothers." Western Journal of Nursing Research 27(6): 676-693. https://doi.org/10.1177/0193945905277154

Hipgrave, D. B., F. Assefa, A. Winoto and S. Sukotjo (2012). "Donated breast milk substitutes and incidence of diarrhoea among infants and young children after the May 2006 earthquake in Yogyakarta and Central Java." Public Health Nutrition 15(2): 307-315. doi: 10.1017/S1368980010003423

IFE Core Group (2015). "Interim Operational Considerations for the feeding support of Infants and Young Children under 2 years of age in refugee and migrant transit settings in Europe."

https://www.ennonline.net/interimconsiderationsiycftransit

IFE Core Group (2017). Infant and Young Child Feeding in Emergencies: Operational Guidance for Emergency Relief Staff and Program Managers, Version $\underline{3}$. https://reliefweb.int/report/world/infant-and-young-child-feedingemergencies-operational-guidance-emergency-relief-0

IRIN (2009). Pakistan: Baby formula risk for IDPs. AlertNet. London, Thomas Reuters Foundation. 2009. http://www.irinnews.org/report/84898/pakistanbaby-formula-risk-idps

Jayathilaka, A. (2005). Sri Lanka. White Ribbon Alliance Bali Conference Special Plenary Session on Maternal Health in Times of Crisis. Bali, Indonesia.

Mboya, S. (2014). "Artificial feeding in emergencies: Experiences from the ongoing Syrian crisis." Field Exchange 48: 164.

https://www.ennonline.net/fex/48/artificialfeeding

Modigell, I., C. Fernandes and M. Gayford (2016). "Save the Children's IYCF-E Rapid Response in Croatia." Field Exchange 52(106-108).

https://www.ennonline.net/fex/52/rapidresponseincroatia

New Zealand Ministry of Health (2012). Food and Nutrition Guidelines for Healthy Infants and Toddlers (Aged 0-2): A Background Paper. Wellington, Ministry of Health. https://www.health.govt.nz/system/files/documents/publications/food-andnutrition-guidelines-healthy-infants-and-toddlers-revised-dec12.pdf 
NHMRC (2013). Infant Feeding Guidelines for Health Workers. Canberra, Commonwealth of Australia.

https://www.nhmrc.gov.au/_files_nhmrc/file/publications/170131_n56_infant_f eeding_guidelines.pdf

Nybo, T. (2010). "'Baby tents' offer quake-affected Haitian mothers a safe place to breastfeed." http://www.unicef.org/infobycountry/haiti_52797.html.

Patel, A., S. Bucher, Y. Pusdekar, F. Esamai, N. F. Krebs, S. S. Goudar, E. Chomba, A. Garces, O. Pasha, S. Saleem, B. S. Kodkany, E. A. Liechty, B. Kodkany, R. J. Derman, W. A. Carlo, K. M. Hambidge, R. L. Goldenberg, F. Althabe, M. Berrueta, J. L. Moore, E. M. McClure, M. Koso-Thomas and P. L. Hibberd (2015). "Rates and determinants of early initiation of breastfeeding and exclusive breast feeding at 42 days postnatal in six low and middle-income countries: A prospective cohort study." Reproductive Health 12(2): S10. doi: 10.1186/1742-4755-12-S2-S10

Prentice, A. M., S. B. Roberts, A. Prentice, A. A. Paul, M. Watkinson, A. A. Watkinson and R. G. Whitehead (1983). "Dietary supplementation of lactating Gambian women. I. Effect on breast-milk volume and quality." Human Nutrition Clinical Nutrition 37(1): 53-64.

Pries, A. M., S. L. Huffman, K. Mengkheang, H. Kroeun, M. Champeny, M. Roberts and E. Zehner (2016). "Pervasive promotion of breastmilk substitutes in Phnom Penh, Cambodia, and high usage by mothers for infant and young child feeding." Maternal \& Child Nutrition 12: 38-51. doi: 10.1111/mcn.12271.

Redmond, E. C. and C. J. Griffith (2009). "The importance of hygiene in the domestic kitchen: implications for preparation and storage of food and infant formula." Perspectives in Public Health 129(2): 69-76.

DOI:10.1177/1757913908101604

Save the Children (n.d.). Main pros and cons of using ready to use infant formula and powdered infant formula. IYCF-E Toolkit: Rapid start-up for emergency nutrition personnel.

Summers, A. and 0. 0. Bilukha (2018). "Suboptimal infant and young child feeding practices among internally displaced persons during conflict in eastern Ukraine." Public Health Nutrition 21(5): 917-926.

https://www.ncbi.nlm.nih.gov/pmc/articles/PMC5848760/

Tarrant, M., K. Y. W. Lok, D. Y. T. Fong, K. M. Wu, I. L. Y. Lee, A. Sham, C. Lam, D. L. Bai, K. L. Wong, E. M. Y. Wong, N. P. T. Chan and J. E. Dodgson (2016). "Effect on baby-friendly hospital steps when hospitals implement a policy to pay for infant formula." Џournal of Human Lactation 32(2): 238-249.

https://doi.org/10.1177/0890334415599399

The Sphere Project (2011). The Sphere Handbook: Humanitarian Charter and Minimum Standards in Disaster Response. Geneva, The Sphere Project. 
UNHCR and Save the Children (2017). Infant and Young Child Feeding in Refugee Situations: A Multi-Sectoral Framework for Action.

http://www.unhcr.org/5acc812e7.pdf

UNICEF. (2008). "Calmness and love for displaced breast feeding mothers in the new temporary shelter." from

http://www.unicef.org/georgia/reallives_10695.html.

UNICEF (2008). Nearly 40000 children in need of urgent assistance in Georgia. ReliebWeb. Kobe, United Nations Office for the Coordination of Humanitarian Affairs. https://www.unicef.org/media/media_45310.html

UNICEF (2013). Breastfeeding on the Worldwide Agenda. New York, UNICEF. https://www.unicef.org/eapro/breastfeeding-worldwide-ExecutiveSummary.pdf

UNICEF (2017). "Joint statement from the Jordan cross border Nutrition working group - Nutrition stakeholders call for appropriate feeding of infants and young children in Southern Syria." https://reliefweb.int/report/syrian-arabrepublic/joint-statement-jordan-cross-border-nutrition-working-groupnutrition

UNICEF, UNHCR, World Health Organization, Save the Children and Emergency Nutrition Network (2015). Interim Operational Considerations for the feeding support of infants and young children under 2 years of age in refugee and migrant transit settings in Europe. https://www.ennonline.net/interimconsiderationsiycftransit

World Health Organization (1981). International Code of Marketing of Breastmilk Substitutes Geneva, World Health Organization. http://www.who.int/nutrition/publications/code_english.pdf

World Health Organization (2005). Guiding Principles for Feeding Non-Breastfed Children 6-24 Months of Age. Geneva, World Health Organization. http://www.who.int/maternal_child_adolescent/documents/9241593431/en/

World Health Organization (2013). "Information concerning the use and marketing of follow-up formula."

http://www.who.int/nutrition/topics/WHO_brief_fufandcode_post_17July.pdf.

World Health Organization and FAO (2007). How to Prepare Formula for CupFeeding at Home. Geneva, World Health Organization. http://www.who.int/foodsafety/document_centre/PIF_Cup_en.pdf

World Health Organization and UNICEF (2003). Global Strategy for Infant and Young Child Feeding. Geneva, World Health Organization. http://www.who.int/nutrition/publications/infantfeeding/9241562218/en/ 
World Health Assembly (1986). "Infant and young child feeding." WHA 39.28. http://www.who.int/nutrition/topics/WHA39.28_iycn_en.pdf?ua=1

World Health Organization (2004). Guiding Principles for Infant and Young Child Feeding in Emergencies. Geneva, World Health Organization. http://www.who.int/nutrition/publications/emergencies/9241546069/en/

World Health Organization (2016). Guideline: Updates on HIV and Infant Feeding. Geneva, World Health Organization.

World Health Organization and UNICEF (2018). HIV and Infant Feeding in Emergencies Operational Guidance. Geneva, World Health Organization. http://www.who.int/nutrition/publications/hivaids/hiv-if-emergenciesguidance/en/

World Health Organization, UNICEF and International Organization for Migration. (2000). "Health Assessment of Internally Displaced Population from Chechnya in Ingushetia 31 January- 5 February 2000."

http://www.reliefweb.int/rw/RWFiles2000.nsf/FilesByRWDocUnidFilename/A COS-64CE6S-chechnyareport8feb.pdf/\$File/chechnyareport8feb.pdf.

World Health Organization, UNAIDS, UNFPA and UNICEF (2010) Guidelines on HIV and Infant Feeding. Principles and Recommendations for Infant Feeding in the Context of HIV and a Summary of Evidence. Geneva, World Health Organization. http://www.who.int/nutrition/publications/hivaids/hiv-ifemergencies-guidance/en/ 